\title{
Polo-Like Kinase 1: A Novel Target for the Treatment of Therapy-Resistant Mantle Cell Lymphoma
}

\author{
Adam K. Ahrens, ${ }^{1}$ Nagendra K. Chaturvedi, ${ }^{1}$ Ashima Shukla, ${ }^{1}$ Tara M. Nordgren, \\ Ganapati V. Hegde, ${ }^{1}$ Julie M. Vose, ${ }^{2}$ and Shantaram S. Joshi ${ }^{1}$ \\ ${ }^{1}$ Department of Genetics, Cell Biology and Anatomy, University of Nebraska Medical Center, \\ 986395 Nebraska Medical Center, Omaha, NE 68198-6395, USA \\ ${ }^{2}$ Department of Internal Medicine, University of Nebraska Medical Center, Omaha, NE 68198-7680, USA
}

Correspondence should be addressed to Shantaram S. Joshi; ssjoshi@unmc.edu

Received 27 September 2012; Revised 29 November 2012; Accepted 16 December 2012

Academic Editor: Umberto Vitolo

Copyright (C) 2013 Adam K. Ahrens et al. This is an open access article distributed under the Creative Commons Attribution License, which permits unrestricted use, distribution, and reproduction in any medium, provided the original work is properly cited.

\begin{abstract}
Mantle cell lymphoma (MCL) is a B-cell non-Hodgkin lymphoma (NHL) which is one of the most aggressive lymphomas. Despite recent improvements in therapies, the development of therapy-resistance is still a major problem; therefore, in order to understand the molecular basis of therapy-resistance, stable therapy-resistant MCL cell lines have been established by us. Based on the gene expression profiles of these cell lines, Polo-like kinase 1 (PLK1) was chosen as a therapeutic target. In this paper, we demonstrate a significant antilymphoma effect of targeting PLK1 in therapy-resistant MCL cells and primary MCL cells from refractory patients. PLK1 knockdown with the antisense oligonucleotide (ASO)/or small molecule inhibitor BI2536 showed significantly decreased proliferation and increased apoptosis in therapy-resistant MCL cell lines and MCL primary cells. Additionally, the direct proteinprotein interaction partners of PLK1 were mapped using ingenuity pathway and confirmed the level of association of these partners with PLK1 based on their expression changes following PLK1 knockdown using real-time PCR. Results suggest that PLK1 is a viable target for the treatment of therapy-resistant MCL.
\end{abstract}

\section{Introduction}

Mantle cell lymphoma (MCL) is an aggressive form of Bcell non-Hodgkin lymphoma. MCL is typically diagnosed at late stage, predominantly in elderly males and commonly metastasizes to multiple sites including the liver, kidney, gastrointestinal tract, or bone marrow [1,2]. Cytogenetically, the majority of MCL patients have a $\mathrm{t}(11 ; 14)(\mathrm{q} 13 ; \mathrm{q} 32)$ which deregulates cyclin D1 (CCND1) and immunoglobulin heavy chain (IGH), resulting in overexpression of CCND1 and increased proliferation $[3,4]$.

While the median overall survival has improved to around seven years with the advancements in therapies, the recurrence due to therapy-resistance is still a major problem in refractory MCL. Combination chemotherapy regimens like CHOP combined with rituximab are common and frontline responses are generally good, but the development of therapy-resistance precludes long-term survival [5-7].
Additional options include high-dose therapy followed by hematopoietic stem cell transplantations and targeted treatments such as bortezomib, lenalidomide, or temsirolimus. However, the resistance ultimately arises even with secondline therapies $[8-10]$. Therefore, it would be beneficial to identify the molecular mechanisms of therapy-resistance in MCL and develop a more specific targeted genetic approach to overcome the problem.

As previously described, our lab has generated therapyresistant MCL cell lines from the relapsed lymphoma from liver (GRL), kidney (GRK), and lungs (GRR) of CHOP and bortezomib-treated NOD-SCID mice bearing Granta 519 human MCL [11, 12]. During the characterization of these therapy-resistant MCL cell lines, gene expression profiling was done in comparison to parental Granta 519 (GP). From these profiles, genes with known function in drug resistance pathways were investigated. PLK1 was selected for further analysis based upon its overexpression in therapy-resistant 
MCL; the fact that a role for PLK1 has not yet been reported in MCL and because overexpression of PLK1 has been reported in other cancers as a poor prognostic marker [13-17]. PLK1 is a kinase that is involved in multiple steps of mitotic progression including CDK1/Cyclin B1 activation, centrosome maturation, chromosomal separation, APC activation, spindle formation, and the regulation of spindle assembly checkpoint control [14, 16-20]. The PLK family was first discovered in drosophila melanogaster $[20,21]$. Four mammalian PLKs have since been discovered (PLK1-4) [21-23].

Antimitotic drugs have been successful in the treatment of various types of cancer $[24,25]$. They are limited by both resistance and toxicity because they affect both normal and cancer cells. Small molecular weight inhibitors of PLK1 and other mitotic genes have begun to be developed and tested in clinical trials in an effort to overcome these problems [26-28]. BI2536 is a small molecule inhibitor of PLK1 and was chosen for use in our studies because it displays remarkably potent and selective inhibition of PLK1. BI2536 was also shown to have anticancer effects in a panel of over 30 different types of cancer cell lines [29-31]. Clinical trials of drugs targeting PLK1 have reported only moderate side effects [29, 30]. Thus, our preliminary results and published reports indicate that PLK1 is a viable target to treat refractory MCL.

We hypothesized that PLK1 is involved with the development of therapy-resistance in MCL and that the knockdown of this gene will effectively kill MCL cells that are resistant to standard therapies. The main objective of this study was to show that PLK1 plays an important role in therapyresistant MCL and that its knockdown has antilymphoma effects in in vitro therapy-resistant cells as well as primary cells of leukemic phase MCL patients. Effective induction of cell death and decreased proliferation in therapy-resistant MCL cells was observed when these cells were treated with low nanomolar concentrations of BI2536, a small molecule inhibitor of PLK1.

\section{Materials and Methods}

2.1. Generation of Therapy-Resistant MCL Cell Lines. The therapy-resistant MCL cell lines were established from the relapsed lymphoma cells from liver, kidney, and lungs from Granta 519 bearing NOD-SCID mice following treatment with CHOP chemotherapy and bortezomib as described previously $[11,12]$. These therapy-resistant tumor cells derived from liver, kidney, and lungs were described as GRL isolated from liver, GRK isolated from kidney, and GRR isolated from lungs, respectively, and compared to parental Granta 519 cells (GP).

2.2. Maintenance of Therapy-Resistant MCL Cells. The parental MCL cell line GP was purchased from Deutsche Sammlung von Mikroorganismen und Zellkulturen (DSMZ, Germany). The parental GP, GRL, GRK, and GRR therapyresistant MCL cell lines were maintained in RPMI media (Invitrogen, CA, USA) containing 10\% FBS (Atlanta Biologicals, GA, USA), $100 \mathrm{U} / \mathrm{mL}$ penicillin, $100 \mu \mathrm{g} / \mathrm{mL}$ streptomycin (Invitrogen, CA, USA) and $2 \mathrm{mM} \mathrm{L-Glutamine}$
(Invitrogen, CA, USA). This medium with supplements is referred to as RF-10 medium in this paper. The cultures were maintained in a humidified incubator adjusted at $5 \% \mathrm{CO}_{2}$ and $95 \%$ air atmosphere at $37^{\circ} \mathrm{C}$. All cultures were passaged twice weekly and maintained at concentration no greater than $1 \times 10^{6} / \mathrm{mL}$.

2.3. Downregulation of PLK1 in Lymphoma Cells. The PLK1 expression was knocked down using antisense oligonucleotide (ASO) or small molecule inhibitor BI2536. The concentration of PLK1 ASO used in the cell treatments was $5 \mu \mathrm{M}$ in each case and the concentration of BI2536 used was $10 \mathrm{nM}$. BI2536 concentration was determined to be the most effective in previous experiments $[30,31]$. In each experiment where ASO was used, an extra control, scrambled (SCR) ASO was also used at $5 \mu \mathrm{M}$ in addition to the untreated controls. The PLK1 and SCR ASO sequences are summarized in Table 1. The knockdown efficiency of both ASO and BI2536 was tested using quantitative real-time PCR. In each case, PLK1 was undetectable in the treated groups after 40 cycles and detectable in untreated groups after approximately 24 cycles.

2.4. In Vitro Growth Assays. Twenty thousand cells from GP and each type of therapy-resistant cell line (GRK, GRL, and GRR) were cultured in triplicate in RF-10 media containing PLK1 inhibitor BI2536 or DMSO (vehicle) in 96-well plates. Similarly, these cells were also treated with PLK1 ASO or SCR ASO along with untreated controls. The growth of these cells were determined at 48 and 72 hours using MTT and 3[H]-thymidine uptake assays. In brief, $25 \mu \mathrm{L}$ of MTT [3-(4,5-dimethylthiazol-2-yl)-2,5-diphenyltetrazolium bromide] reagent ( $5 \mathrm{mg} / \mathrm{mL}$ in PBS) was added to the culture and incubated for two hours before the respective time point, and the cells were lysed using an SDS-based lysing reagent. The intensity of the color developed was measured at $570 \mathrm{~nm}$ using a plate reader (BioRad). In another set of experiments, $0.5 \mu \mathrm{Ci}$ of $3[\mathrm{H}]$-thymidine was added 18 hours prior to cell harvest. The cells were harvested at 48 and 72 hours using a PHD cell harvester (Cambridge Technologies, MA, USA). The incorporated radioactivity was counted using a liquid scintillation counter (Packard Instruments, IL, USA).

2.5. PLK1 ASO Withdrawal Assay. Twenty thousand cells from each type of therapy-resistant cell line (GRL, GRK, and GRR) and parental GP were cultured in triplicate in RF-10 media containing PLK1 ASO or scrambled ASO (control) in 96-well plates. After 48 hours of the ASO treatment, withdrawal of PLK ASO was performed in the culture following spin down of the cells and then fresh RF-10 media was added to the cells. Following withdrawal, cells were further cultured for 48- and 96-hour time points and their viability was determined using MTT growth assay. The viability of cells was compared with PLK ASO-silenced or control ASOtreated cells.

2.6. Apoptosis and Cytomorphological Analyses. The MCL cells were cultured at a concentration of $1 \times 10^{6}$ cells $/ \mathrm{mL}$ in RF-10 media containing BI2536 or DMSO or PLK1 ASO 
TABLE 1: List of primers and sequences of antisense oligonucleotide (F: forward, R: reverse).

\begin{tabular}{lcc}
\hline Name of gene & Genebank accession & $\begin{array}{c}\text { Sequence } \\
5^{\prime} \rightarrow 3^{\prime}\end{array}$ \\
\hline $\begin{array}{l}\text { PSMB5 (F) } \\
\text { PSMB5 (R) }\end{array}$ & NM_002797 & AGGCGTGCTTGCCAGCAGTC \\
PKMYT (F) & NM_004203 & GATAGGCCGGGCAACGCCTC \\
PKMYT (R) & & GTCTGe \\
CHUK (F) & NM_001278 & GACCGTGTGCTCAAGGAGCTGT \\
CHUK (R) & AM_001786 & ACAAGGGACCGGGCAGAACTCT \\
CDK1 (F) & TGGCCTTGCCAGAGCTTTTGGA \\
CDK1 (R) & NM_002392 & TGAGTAACGAGCTGACCCCAGCA \\
MDM2 (F) & TTTCGCAGCCAGGAGCACCG \\
MDM2 (R) & AM_003600 & AGTTTCCTTCACGGGGCGCG \\
AURKA (F) & TCGGTTCCTCCGTCCCTGAGTGTC \\
AURKA (R) & TCGGTCCATGATGCCTCTAGCTGT \\
PLK1 antisense (ASO) & NM_005030 & ACCAGTCCGGAGGGGAGGGC \\
Scrambled antisense (ASO) & & CAGGGCTGACAGCGAGGCGG \\
\hline
\end{tabular}

or SCR ASO for the desired time points. Following the treatment, the percentage of cells undergoing apoptosis was determined using Annexin-V assay kit (BD Biosciences, CA, USA). Briefly, the cells were washed twice with cold PBS and resuspended in $1 \mathrm{X}$ binding buffer; $5 \mu \mathrm{L}$ of Annexin-VFITC and $2 \mu \mathrm{L}$ of propidium iodide (PI) were added and allowed to incubate in room temperature in the dark for 15 minutes; $400 \mu \mathrm{L}$ of additional $1 \mathrm{X}$ binding buffer was added to each sample, and then the percent of the cells $($ Annexin-V+) undergoing apoptosis was assessed by flow cytometry immediately. For the cytomorphological analyses in treated and untreated cells, cytospin preparations were made from different MCL cells used and stained with WrightGiemsa stain in the UNMC pathology core lab, and then the cytomorphology was examined using a light microscopy.

2.7. Primary MCL Cells from Patients. Primary MCL cells were obtained from leukemic phase MCL patients using an institutional review board approved protocol and informed consent. Mononuclear cells (MNCs) were isolated from MCL patient peripheral blood using lymphocyte separation medium (Accurate Chemical and Scientific, Westbury, NY) described previously [32] and maintained in RF-10 medium. The immunophenotypes of the MNCs were determined by flow cytometry using CD5-PE and CD20-FITC antibodies (BD Biosciences, CA, USA). The proportion of double positive (CD5+CD20) cells was determined using a flow cytometer according to Becton Dickinson manufacturer's instructions (BD Biosciences, CA, USA). Cells having $>90 \%$ double positive for CD5 and CD20 were treated with vehicle control (DMSO) or BI2536 for 24, 48, and 72 hours, and then cells were subjected for the analyses of cell growth and apoptosis using MTT/3[H]-thymidine uptake and annexin-V assays, respectively.

2.8. Quantitative Real-Time PCR. GRK, GRL, GRR, and parental GP cells were cultured in RF-10 media containing BI2536 or vehicle (DMSO) treatment. Following 24 hours of incubation, total RNA from each group was extracted and purified using TRI Reagent according to the manufacturer's instructions (Invitrogen, CA, USA). Five micrograms of total RNA was then used for reverse transcription with the superscript RT enzyme (Invitrogen, CA, USA). The resulting cDNA was subjected to SYBR green real-time PCR to investigate the differential expression of PLK1 interacting genes including AURKA, PSMB5, MDM2, PKMYT, CHUK, and CDK1. The primer sequences used for these genes are summarized in Table 1 . The cDNA, gene primers, and SYBR green PCR master mix (Applied Biosystems, CA, USA) were combined, and real-time PCR was performed using the ABI Prism-7900HT real-time PCR detection system (Applied Biosystems, CA, USA). The PCR reaction consisted of 36 cycles of 30 seconds at $95^{\circ} \mathrm{C}$, then 30 seconds at $60^{\circ} \mathrm{C}$, and finally 30 seconds at $72^{\circ} \mathrm{C}$. Cycle threshold $(\mathrm{Ct})$ values were used to determine fold changes in expression and compared among treatment groups after being normalized with GAPDH.

\section{Results}

3.1. Targeting PLK1 Leads to Decreased Cell Growth and Increased Apoptosis in Refractory MCL. Since the PLK1 is overexpressed in refractory MCL and PLK1 expression has shown to be associated with poor clinical outcome in other cancers, we targeted the PLK1 expression in therapyresistant MCL cells. For the initial experiments we used the specific antisense oligonucleotide (ASO) to downregulate PLK1 expression. Subsequently, we have used BI2536, a small molecule inhibitor of PLK1 to inhibit expression of PLK1, in therapy-resistant MCL cells.

In order to determine the effect of downregulation of PLK1 in therapy-resistant MCL cells, proliferation and survival were measured using MTT assay, 3[H]-thymidine uptake method and annexin-V staining, respectively. Figure 1 shows the effect of downregulation of PLK1 expression using ASO or BI2536, the inhibitors of PLK1 expression. 


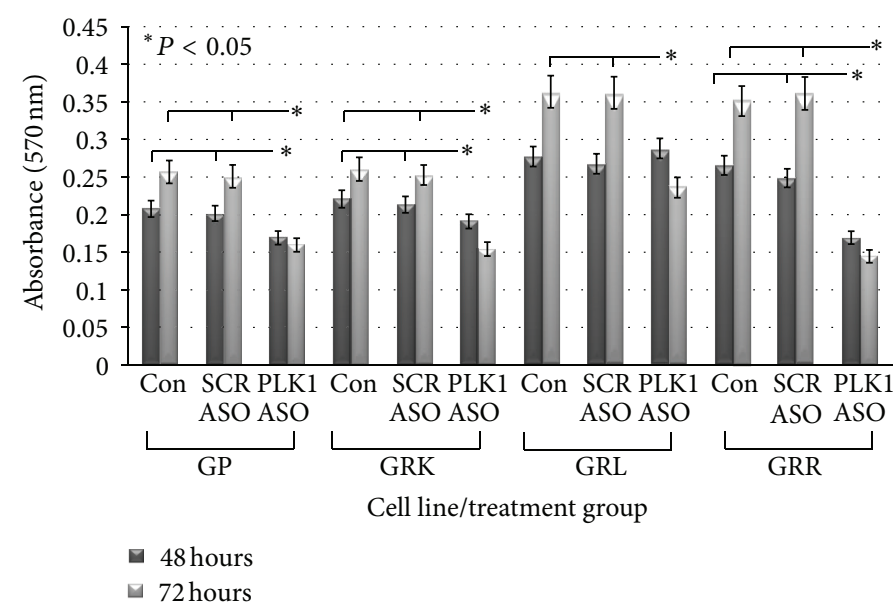

(a)

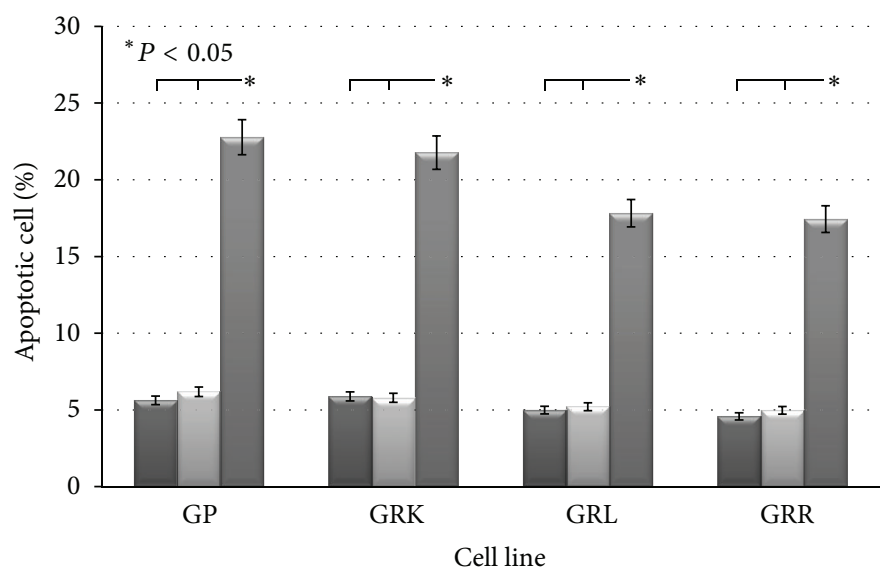

- Cells only control

$\square$ SCR ASO

• PLK1 ASO

(c)

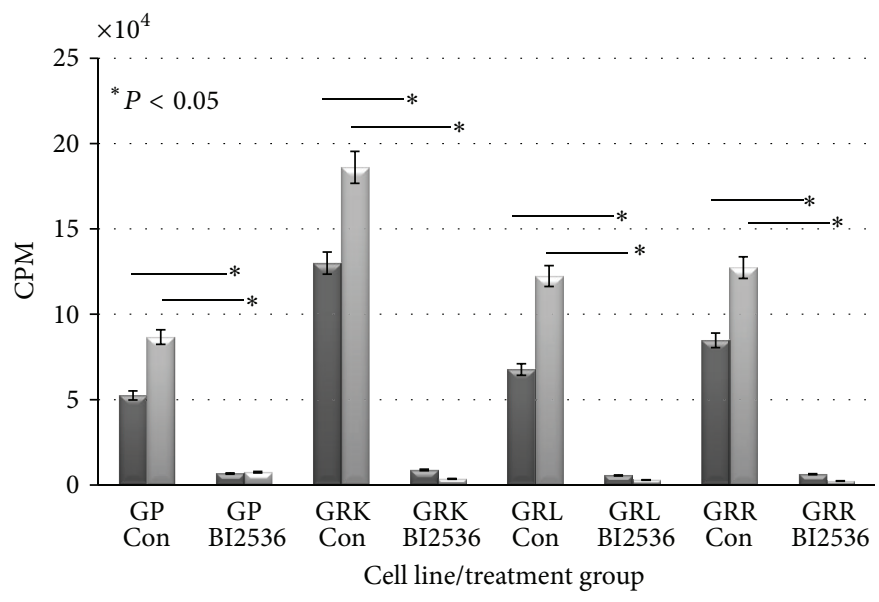

- 48 hours

- 72 hours

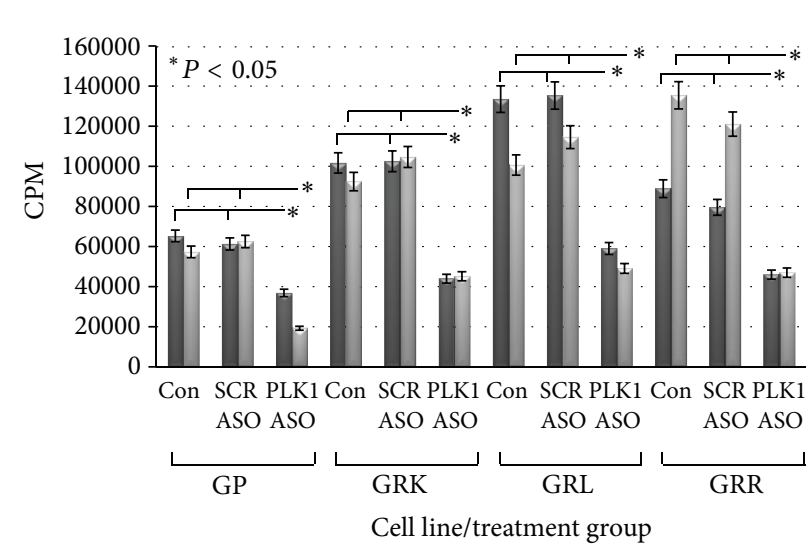

- 48 hours

72 hours

(b)

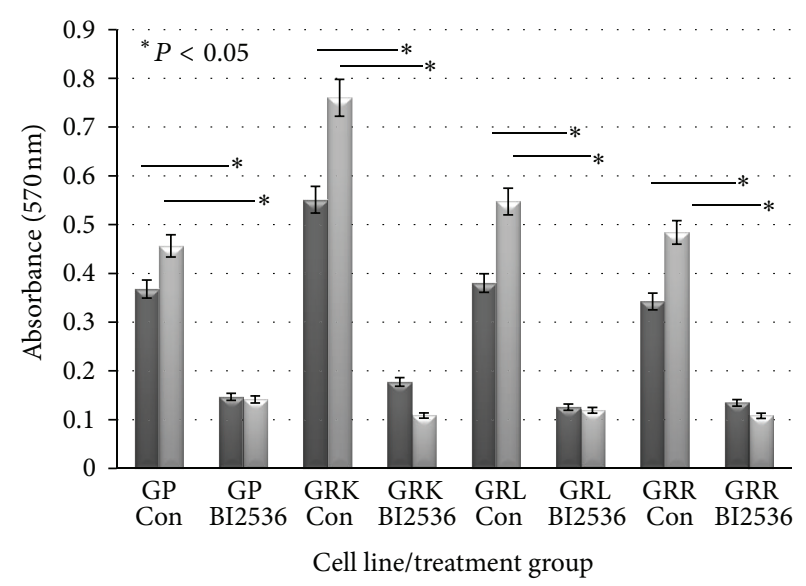

- 48 hours

๘ 72 hours

(d)

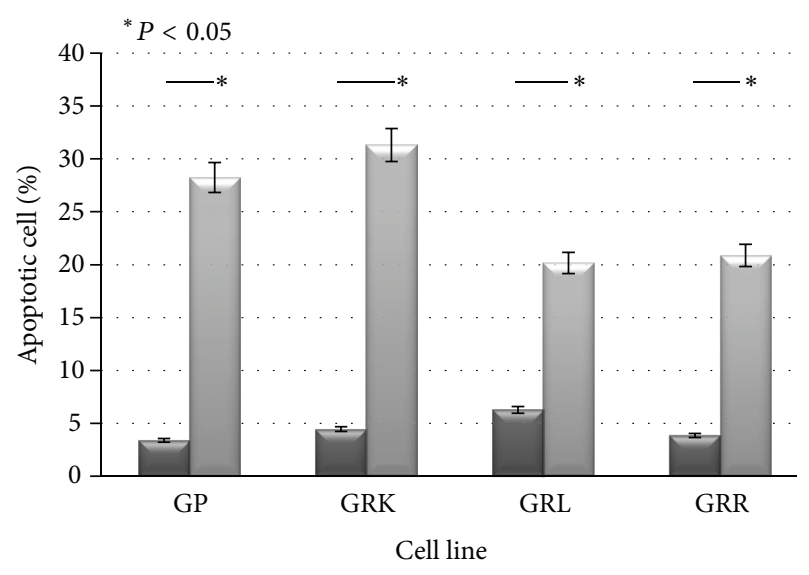

- Cells only control

ॠ BI2536

(e)

Figure 1: Continued. 


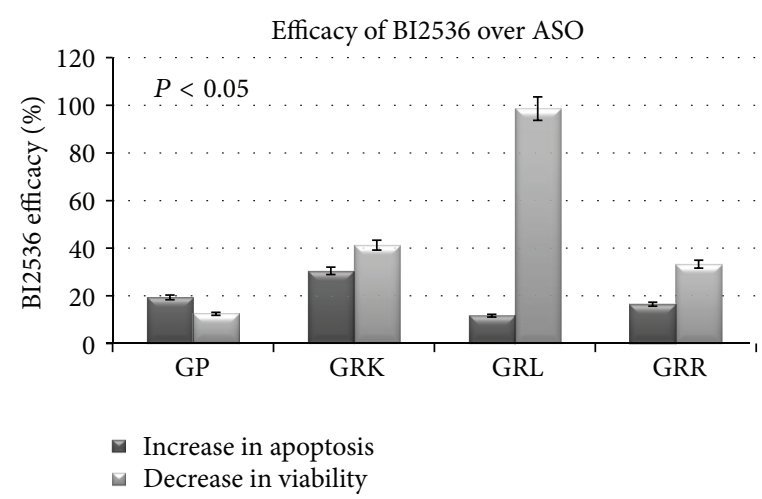

(g)

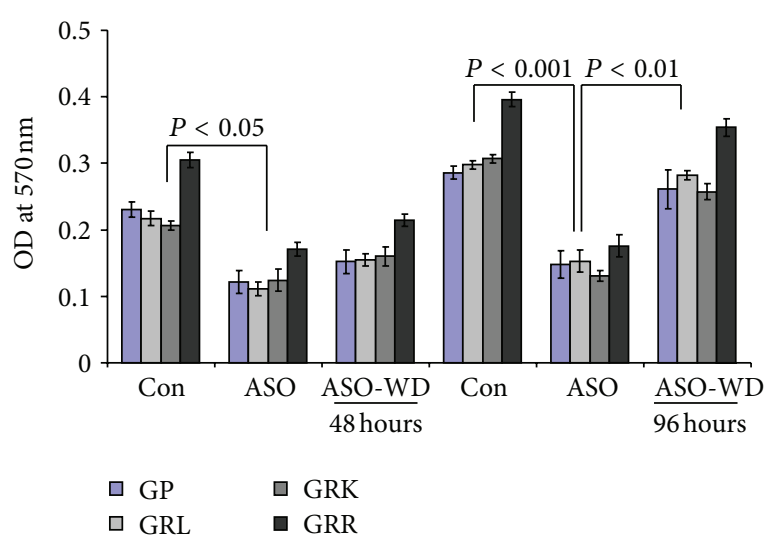

(h)

FIGURE 1: In vitro viability, proliferation, and apoptosis following PLK1 downregulation using BI2536/ASO. Figure 1 shows the changes to the level of viability, proliferation, and apoptosis in each of the therapy-resistant cell lines and parental GP following PLK1 downregulation with 48 and 72 hours treatment with either ASO ((a)-(c)) or BI2536 ((d)-(f)). In case of PLK1 ASO treatment, untreated/scrambled (SCR) ASO while in BI2536 treatment, vehicle- (DMSO-) treated cells were used as control groups for comparison in these experiments. (g) Represents the efficacy of BI2536 over ASO treatment in decreasing cell viability and increasing apoptosis in therapy-resistant and parental MCL cells. (h) MTT assay showing the restoration of the viability in therapy-resistant and parental MCL cells after PLK1 reintroduction by PLK1 ASO withdrawal (ASO-WD) for 48 and 96 hours following PLK1 silencing for 48 hours. SCR ASO (Con) was used as a control in this experiment. In these experiments, the values represent the means \pm SE from triplicate wells of the 96-well plates. Similar results were obtained from two sets of independent experiments. ${ }^{*} P$ indicates the statistical difference between control MCL cells and PLK1 downregulated MCL cells.

Figures 1(a)-1(c) show MCL cell viability, proliferation, and apoptosis when PLK1 has been knocked down with ASO. The viability and proliferation of each of the therapy-resistant cells were significantly decreased (Figures 1(a) and 1(b)) following PLK1 downregulation using ASO. Such a decrease in cell viability and proliferation was not observed in MCL cells treated with SCR ASO or untreated control cells (Figures 1(a) and 1(b)). Correspondingly, the apoptosis levels were significantly increased in each of the therapy-resistant MCL cells following targeting PLK1 compared to SCR ASO or untreated control cells (Figure 1(c)). Similarly, therapy-resistant MCL cells treated with BI2536, the inhibitor of PLK1 expression, also showed a very significant decrease in the viability and decrease in proliferation of these cells compared to cells treated with vehicle- (DMSO-) treated control cells (Figures $1(\mathrm{~d})$ and $1(\mathrm{e})$ ). Furthermore, there was a very significant increase in the percentage of MCL cells undergoing apoptosis following downregulation of PLK1 as determined by annexin$\mathrm{V}$ staining compared to control cells (Figure 1(f)). All four cell lines behaved very similarly in each of these experiments suggesting that PLK1 is able to function in both refractory and parental MCL cells. We then compared the efficacy of PLK1 ASO versus BI2536, the PLK1 inhibitor. The efficacy of the BI2536 was significantly $(P<0.05)$ superior compared to PLK1 ASO. Figure 1(g) shows the efficacy of BI2536 over PLK1 ASO, clearly demonstrating the better targeting of PLK1.

We also investigated whether PLK1 reintroduction restores the chemoresistant properties in the PLK1 ASOsilenced therapy-resistant and parental GP MCL cells. For this purpose, we performed PLK1 ASO withdrawal experiment after PLK1 silencing in therapy-resistant and parental GP MCL cell lines that can restore the expression of PLK1. Our results clearly showed that following 96 hours PLK1 ASO withdrawal/depletion, the viability of these MCL cell lines was significantly increased compared to the PLK1-silenced cells, as determined by MTT growth assay (Figure 1(h)). In addition, results also showed that the level of viability was very close to the control (SCR ASO) cells after 96 hours PLK1 ASO withdrawal, suggesting that PLK1 reintroduction after silencing restores the growth of MCL cell lines.

In addition to annexin- $\mathrm{V}$ staining to measure the apoptotic cell population, we also examined the MCL cells following downregulation of PLK1 with BI2536 using light microscopy of Giemsa stained MCL cells. As shown in Figure 2, there was a significant increase in apoptotic bodies and fragmented cells in MCL cells treated with BI2536 compared to control MCL cells.

3.2. Effects of PLK1 Downregulation in Primary MCL Cells. Once we confirmed the cytotoxic effects of targeting PLK1 in MCL cell lines, as a logical next step to determine the antilymphoma effects of BI2536 against primary refractory MCL cells, we treated the MCL cells purified from patient blood with leukemic phase of MCL. Figures 3(a) and 3(b) show the viability and proliferation of primary refractory MCL cells following treatment with BI2536, the inhibitor of PLK1, for 24,48 , and 72 hours. There was a significant decrease in the viability and proliferation of refractory primary MCL cells following treatment with BI2536. Furthermore, the treatment of these primary MCL cells with BI2536 also resulted in the significant increase in the apoptosis of primary MCL cells as shown in Figure 3(c). This data further validates the idea that PLK1 knockdown is an effective target in refractory MCL. 

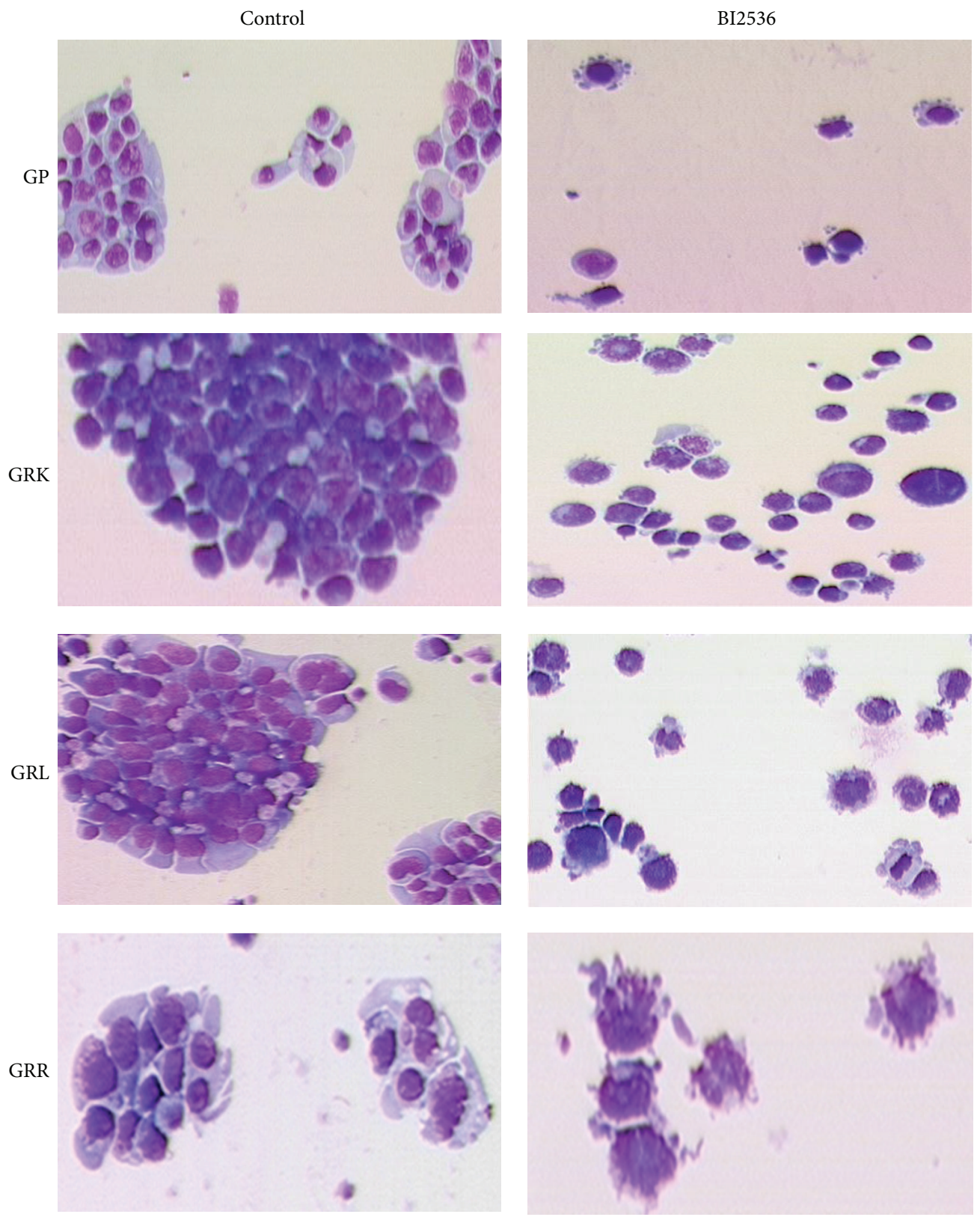

FIgURE 2: Cytomorphology of therapy-resistant MCL cells following PLK1 downregulation. Parental and therapy-resistant MCL cells treated with or without BI2536 inhibitor of PLK1 were evaluated for the presence of apoptotic bodies using cytomorphology. The rows from top to bottom consist of GP, GRK, GRL, and GRR. The left column consists of control- (DMSO-) treated cells and the right column consists of cells that have had PLK1 knocked down with BI2536. These observations were examined 48 hours after treatment at 40x magnification using a light microscopy.

3.3. Molecular Changes Associated with PLK1 Downregulation. PLK1 knockdown produces significant reductions in viability/proliferation and significant increases in apoptosis of clinical patient samples and therapy-resistant MCL cells isolated from different tissue sites. We next wanted to investigate the molecular changes caused by the inhibition of PLK1 expression and how they relate to genes associated with therapy-resistance. Figure 4(a) shows an interaction network featuring PLK1 at the center that was derived using Ingenuity Pathway Analysis (IPA) software. This interaction network was used to identify genes which closely interact with PLK1. Since therapy-resistance in MCL can influence gain or loss of function of a number of genes, we wanted to explore how some of the potent genes behave with PLK1 downregulation to understand the changes that may occur in therapy-resistant MCL following treatment with BI2536. In this regard, initially, nonquantitative RT-PCR was performed to confirm downregulation of PLK1 following treatment with BI2536 in therapy-resistant and parental MCL cells. RTPCR result showed a very low or undetectable expression 


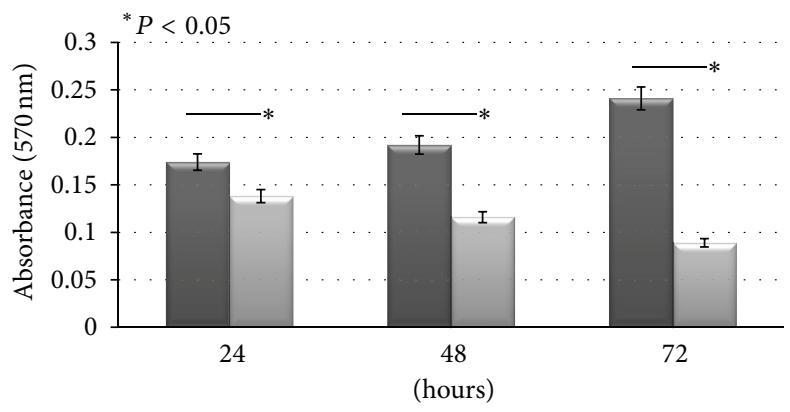

(a)

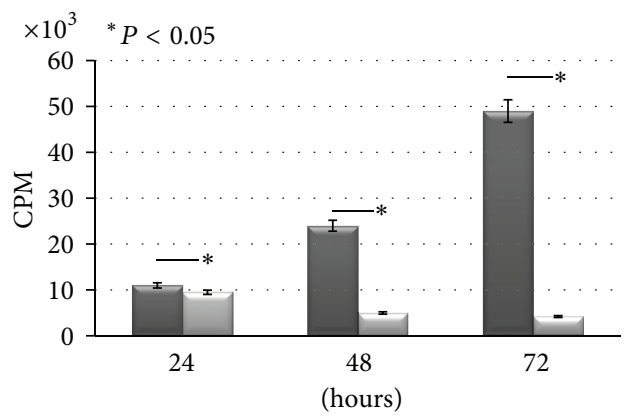

(b)

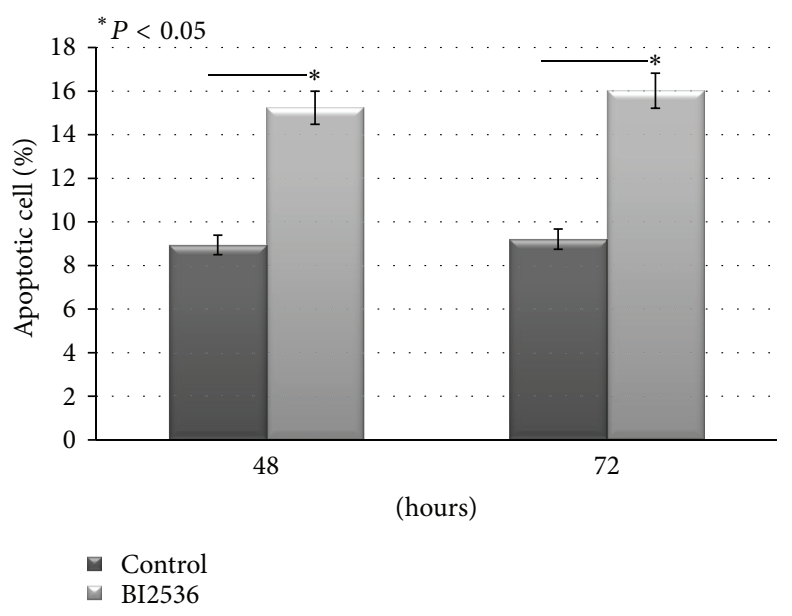

(c)

FIGURE 3: Viability, proliferation, and apoptosis of primary refractory MCL cells following PLK1 downregulation with BI2536. Viability (a) and proliferation (b) were measured at 24, 48, and 72 hours after treatment using MTT and 3[H]-thymidine uptake assays, respectively, while apoptosis (c) was measured at 48 and 72 hours using annexin-V staining. The values represent the means \pm SE from triplicate wells of the 96 well plates. These results are representative from two different MCL patient samples. ${ }^{*} P$ indicates the statistical difference between control primary MCL cells and PLK1 downregulated primary MCL cells.

of PLK1 in BI2536-treated MCL cell lines compared to control. Figure 4(b) shows a downregulation of PLK1 in GRL MCL cells following treatment with BI2536 for 24 hours. Similar downregulation of PLK1 was observed in each therapy-resistant and parental GP MCL cell. Further, real-time PCR was performed following BI2536 treatment of therapy-resistant and parental MCL cells for the genes AURKA, MDM2, CDK1, PSMB5, PKMYT, and CHUK which are summarized in Figure 4(c). The downregulation of PLK1 using BI2536 altered the expression of AURKA, MDM2, PSMB5, CHUK, PKMYT, and CDK1. AURKA is a kinase like PLK1 and is necessary for normal cell division; MDM2 is a negative p53 regulator; PSMB5 is a proteasomal subunit which binds with PLK1; CHUK is an inhibitor NF-kB; PKMYT is a cell cycle inhibitor which is inhibited by PLK1; CDK1 is a positive regulator of cell cycle progression [14, 19, 21, 25]. Following knockdown of PLK1, qRT-PCR results showed the significantly decreased expression of most of the genes mentioned above except PSMB5 and PKMYT in both therapy-resistant and parental GP MCL cells. However, the level of expression of the genes varied among these cells. The underexpressed genes such as AURKA, MDM2, CHUK, and $\mathrm{CDK} 1$, which are known to be positive regulators of PLK1, were well correlated with downregulated PLK1. On the contrary, PKMYT, which is known to be a negative regulator of PLK1, was overexpressed and inversely correlated with downregulated PLK1 in therapy-resistant MCL cells. GRL therapy-resistant MCL was the only cell line to underexpress PSMB5. The results of this experiment show that although there is some variability in the response, AURKA, MDM2, CHUK, and CDK1 expression goes down, while PKMYT goes up, suggesting their involvement in PLK1-mediated regulation of proliferation and survival of MCL.

\section{Discussion}

In this paper, we have demonstrated that targeting mitotic kinases is a viable strategy to treat therapy-resistant MCL cells. Specifically, we have targeted the well-studied and overexpressed member PLK1 of the mitotic kinases in refractory MCL cells. We used both PLK1-specific antisense oligonucleotides and small molecule inhibitor BI2536 of PLK1 to downregulate PLK1 expression. 


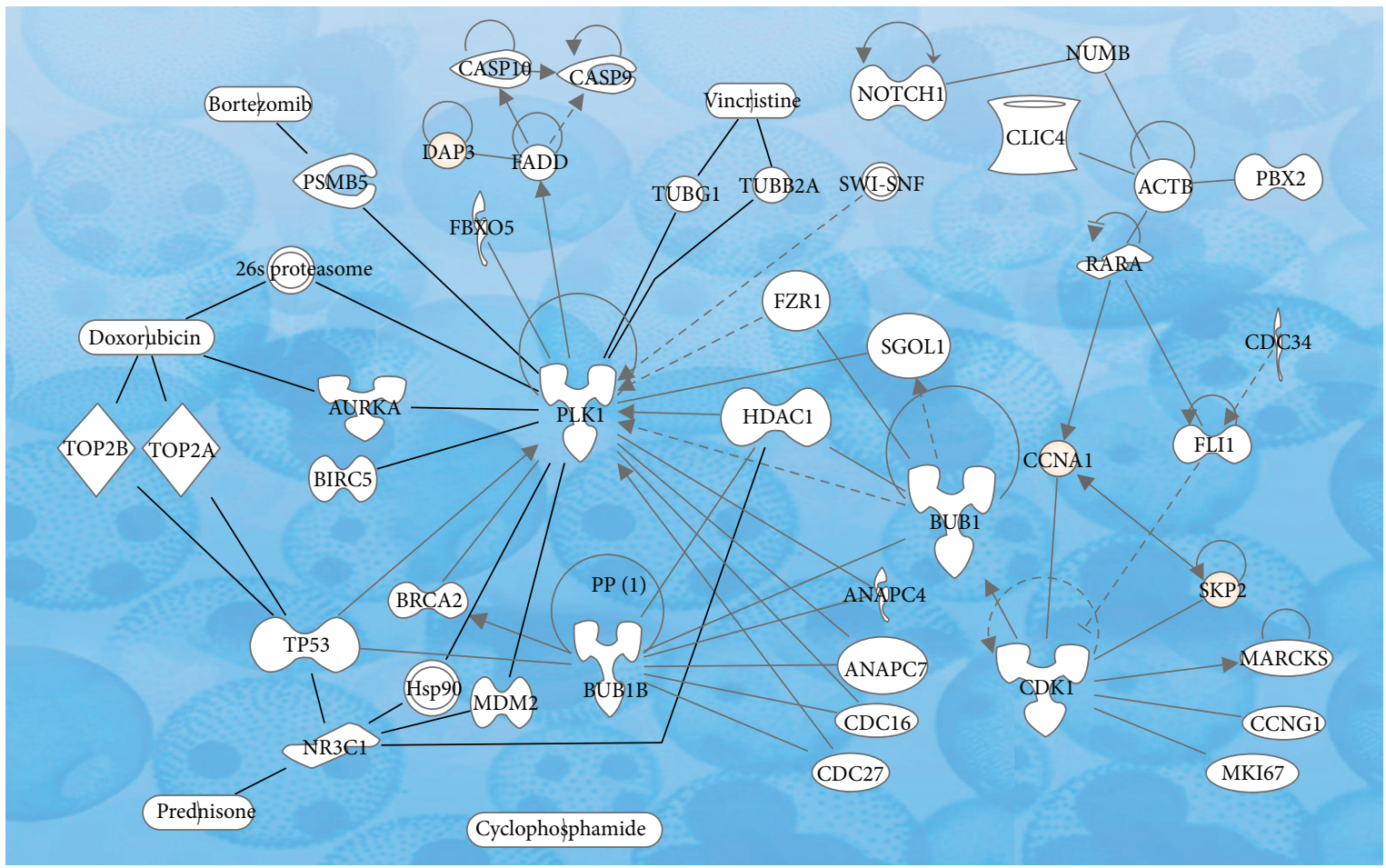

(a)

\begin{tabular}{ll} 
PLK1 mRNA expression & \\
\hline Control & BI2536 \\
& \\
& \\
&
\end{tabular}

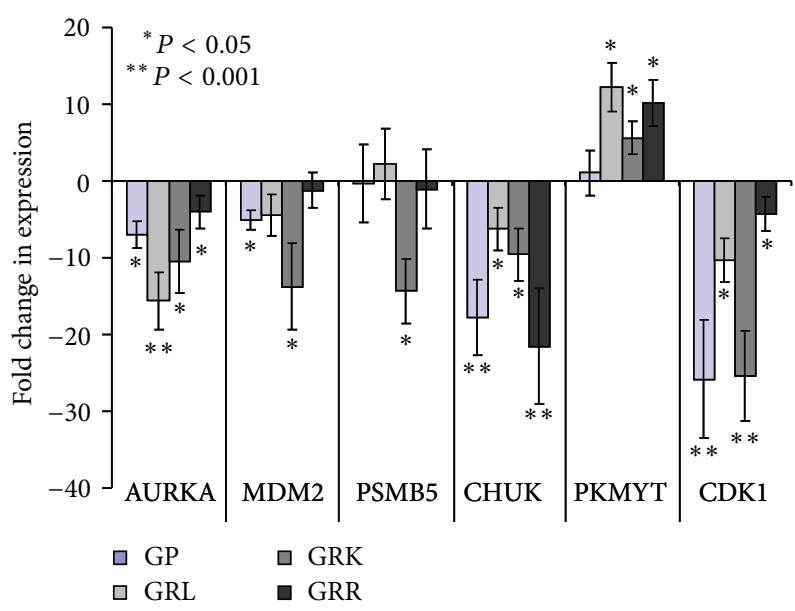

(b)

(c)

FIGURE 4: Analyses of PLK1 interactive proteins and their expression levels following PLK1 downregulation. (a) IPA analyses demonstrate a protein-protein interaction network featuring PLK1 at the center. This network shows some of the direct interactions that take place with PLK1 and which proteins act as intermediates between PLK1 and the drugs used to generate the therapy-resistant cell lines. (b) Represents a nonquantitative RT-PCR result for the expression of PLK1 in GRL MCL cells following 24 hours of treatment with BI2536. (c) Quantitative RTPCR results showing expression of selected PLK1-associated genes from IPA analyses in therapy-resistant and parental MCL cells following downregulation with BI2536. Expression of each gene was analyzed from Ct value obtained from real-time PCR. Fold change of genes expression was calculated with respect to vehicle- (DMSO-) treated control cells and normalized to GAPDH.

Similar to other malignancies, targeting PLK1 resulted in a significant decrease in the proliferation and survival of refractory MCL cells in vitro [14,27-31]. In this study, we have evaluated the effects of both PLK1 ASO and small molecule inhibitor for downregulating the expression of PLK1 in MCL cells. Our finding not only showed decrease in proliferation/survival of MCL cells following PLK1 silencing but also clearly indicated that reintroduction of PLK1 expression restores chemoresistance properties in these cells, suggesting PLK1 involvement in refractory MCL (Figure 1(h)). These 
studies indicated that while ASO inhibited the expression of PLK1 in MCL cells, the extent inhibition and subsequent increase in apoptotic death of MCL cells was significantly higher with the BI2536 inhibitor of PLK1 expression. Although the antitumor activity of BI2536 has been reported [27-31], this paper is the first one to demonstrate its antitumor activity against refractory MCL.

Since our ultimate goal is to take the PLK1 inhibitors to clinical setting in treating MCL patients, we also investigated the effect of PLK1 against primary MCL cells isolated from patients with refractory MCL. These studies demonstrated that BI2536 effectively inhibited the survival and proliferation of primary MCL cells and increased the apoptotic death of these cells.

In addition to demonstrating the antilymphoma activity of BI2536 against refractory MCL, to determine the molecular consequence of downregulating PLK1, we also examined the expression of several genes that interact/associate with PLK1 using quantitative PCR method. Interestingly, PLK1 downregulation resulted in downregulation of AURKA, MDM2, CHUK, and CDK1 which are known to be positive regulators of PLK1 whereas PKMYT, which is a negative regulator of PLK1, was upregulated in PLK1 downregulated therapy-resistant MCL cells suggesting their association with PLK1 downregulation. These results indicate that the robust antitumor effects we have observed might be due to the alteration in the expression of other key molecules that regulate cell division and survival.

In summary, this paper demonstrates that BI2536 is a viable agent to treat refractory MCL. Our results with PLK1 inhibitor against therapy-resistant MCL cells and primary MCL cells, as well as other reports showing its antitumor activity against other cancer, lay the foundation for taking this inhibitor to phase I clinical trial.

\section{Authors' Contribution}

A. K. Ahrens and N. K. Chaturvedi indicate equal contribution to this work as co-first authors.

\section{Acknowledgments}

This work was supported by Lymphoma Research Foundation, New York, NY, USA and partially supported from the UNMC, College of Medicine Dean's Research Funds. The authors also thank Kathryn Hyde for her help in preparing this paper.

\section{References}

[1] F. Bertoni and M. Ponzoni, "The cellular origin of mantle cell lymphoma," International Journal of Biochemistry and Cell Biology, vol. 39, no. 10, pp. 1747-1753, 2007.

[2] H. Nogai, B. Dörken, and G. Lenz, "Pathogenesis of nonHodgkin's lymphoma," Journal of Clinical Oncology, vol. 29, no. 14, pp. 1803-1811, 2011.

[3] P. Pérez-Galán, M. Dreyling, and A. Wiestner, "Mantle cell lymphoma: biology, pathogenesis, and the molecular basis of treatment in the genomic era," Blood, vol. 117, no. 1, pp. 26-38, 2011.

[4] P. Jares, D. Colomer, and E. Campo, "Genetic and molecular pathogenesis of mantle cell lymphoma: perspectives for new targeted therapeutics," Nature Reviews Cancer, vol. 7, no. 10, pp. 750-762, 2007.

[5] J. P. Leonard, M. E. Williams, A. Goy et al., "Mantle cell lymphoma: biological insights and treatment advances," Clinical Lymphoma \& Myeloma, vol. 9, no. 4, pp. 267-277, 2009.

[6] M. E. Williams, M. Dreyling, J. Winter, S. Muneer, and J. P. Leonard, "Management of mantle cell lymphoma: key challenges and next steps," Clinical Lymphoma, Myeloma and Leukemia, vol. 10, no. 5, pp. 336-346, 2010.

[7] M. Dreyling and W. Hiddemann, "Current treatment standards and emerging strategies in mantle cell lymphoma," Hematology, pp. 542-551, 2009.

[8] M. Ghielmini and E. Zucca, "How I treat mantle cell lymphoma," Blood, vol. 114, no. 8, pp. 1469-1476, 2009.

[9] A. M. Gianni, M. Magni, M. Martelli et al., "Long-term remission in mantle cell lymphoma following high-dose sequential chemotherapy and in vivo rituximab-purged stem cell autografting (R-HDS regimen)," Blood, vol. 102, no. 2, pp. 749-755, 2003.

[10] E. Jacobsen and A. Freedman, "An update on the role of highdose therapy with autologous or allogeneic stem cell transplantation in mantle cell lymphoma," Current Opinion in Oncology, vol. 16, no. 2, pp. 106-113, 2004.

[11] A. K. Ahrens, N. K. Chaturvedi, T. M. Nordgren, B. J. Dave, and S. S. Joshi, "Establishment and characterization of therapyresistant mantle cell lymphoma cell lines derived from different tissue sites," Leukemia \& Lymphoma, vol. 53, no. 11, pp. 22692278, 2012.

[12] G. V. Hegde, T. M. Nordgren, C. M. Munger, A. K. Mittal, P. J. Bierman, D. D. Weisenburger et al., "Novel therapy for therapyresistant mantle cell lymphoma: multipronged approach with targeting of hedgehog signaling," International Journal of Cancer, vol. 131, no. 12, pp. 2951-2960, 2012.

[13] F. Barr, H. Sillje, and E. Nigg, "Polo-like kinases and the orchestration of cell division," Nature Reviews Molecular Cell Biology, vol. 5, pp. 429-440, 2004.

[14] K. Strebhardt and A. Ullrich, "Targeting polo-like kinase 1 for cancer therapy," Nature Reviews Cancer, vol. 6, no. 4, pp. 321$330,2006$.

[15] F. Eckerdt, J. Yuan, and K. Strebhardt, "Polo-like kinases and oncogenesis," Oncogene, vol. 24, no. 2, pp. 267-276, 2005.

[16] A. Ahr, T. Karn, C. Solbach et al., "Identification of high risk breast-cancer patients by gene expression profiling," The Lancet, vol. 359, no. 9301, pp. 131-132, 2002.

[17] M. Petronczki, P. Lénárt, and J. M. Peters, "Polo on the rise-from mitotic entry to cytokinesis with Plk1," Developmental Cell, vol. 14, no. 5, pp. 646-659, 2008.

[18] V. Archambault and D. Glover, "Polo-like kinases: conservation and divergence in their functions and regulation," Nature Reviews Molecular Cell Biology, vol. 10, no. 4, pp. 265-275, 2009.

[19] S. M. A. Lens, E. E. Voest, and R. H. Medema, "Shared and separate functions of polo-like kinases and aurora kinases in cancer," Nature Reviews Cancer, vol. 10, no. 12, pp. 825-841, 2010.

[20] B. Fenton and D. M. Glover, "A conserved mitotic kinase active at late anaphase-telophase in syncytial Drosophila embryos," Nature, vol. 363, no. 6430, pp. 637-640, 1993. 
[21] D. M. Glover, I. M. Hagan, and A. Tavares, "Polo-like kinases: a team that plays throughout mitosis," Genes and Development, vol. 12, no. 24, pp. 3777-3787, 1998.

[22] F. J. Clay, S. J. McEwen, I. Bertoncello, A. F. Wilks, and A. R. Dunn, "Identification and cloning of a protein kinase-encoding mouse gene, Plk, related to the polo gene of Drosophila," Proceedings of the National Academy of Sciences of the United States of America, vol. 90, no. 11, pp. 4882-4886, 1993.

[23] U. Holtrich, G. Wolf, A. Bräuninger et al., "Induction and downregulation of PLK, a human serine/threonine kinase expressed in proliferating cells and tumors," Proceedings of the National Academy of Sciences, vol. 91, no. 5, pp. 1736-1740, 1994.

[24] R. Lake and W. Jelinek, "Cell cycle- and terminal differentiationassociated regula-tion of the mouse mRNA encoding a conserved mitotic protein kinase," Molecular and Cellular Biology, vol. 13, no. 12, pp. 7793-7801, 1993.

[25] J. R. Jackson, D. R. Patrick, M. M. Dar, and P. S. Huang, "Targeted anti-mitotic therapies: can we improve on tubulin agents?” Nature Reviews Cancer, vol. 7, no. 2, pp. 107-117, 2007.

[26] K. Strebhardt and A. Ullrich, "Paul Ehrlich's magic bullet concept: 100 years of progress," Nature Reviews Cancer, vol. 8, no. 6, pp. 473-480, 2008.

[27] K. Mross, A. Frost, S. Steinbild et al., "Phase I dose escalation and pharmacokinetic study of BI 2536, a novel polo-like kinase 1 inhibitor, in patients with advanced solid tumors," Journal of Clinical Oncology, vol. 26, no. 34, pp. 5511-5517, 2008.

[28] J. Pawel von, M. Reck, W. Digel et al., "Random-ized phase II trial of two dosing schedules of BI, 2536, a novel Plk-1 inhibitor, in patients with relapsed advanced or metastatic non-small-cell lung cancer (NSCLC)," in ASCO Meeting Abstracts, abstract no. 8030, 2008.

[29] R. Evans, G. Dueck, R. Sidhu et al., "Expression, adverse prognostic significance and therapeutic small molecule inhibition of Polo-like kinase 1 in multiple myeloma," Leukemia Research, vol. 35, no. 12, pp. 1637-1643, 2011.

[30] M. Raab, S. Kappel, A. Krämer et al., "Toxicity modeling of Plk1targeted therapies in genetically engineered mice and cultured primary mammalian cells," Nature Communications, vol. 2, article 395, 2011.

[31] M. Steegmaier, M. Hoffmann, A. Baum et al., "BI 2536, a ootent and selective inhibitor of polo-like kinase 1, inhibits tumor growth in vivo," Current Biology, vol. 17, no. 4, pp. 316-322, 2007.

[32] G. V. Hegde, C. M. Munger, K. Emanuel et al., "Targeting of sonic hedgehog-GLI signaling: a potential strategy to improve therapy for mantle cell lymphoma," Molecular Cancer Therapeutics, vol. 7, no. 6, pp. 1450-1460, 2008. 


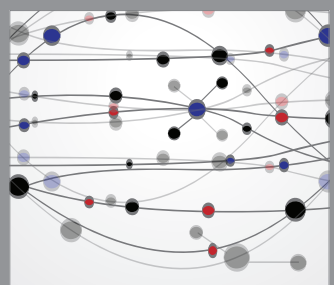

The Scientific World Journal
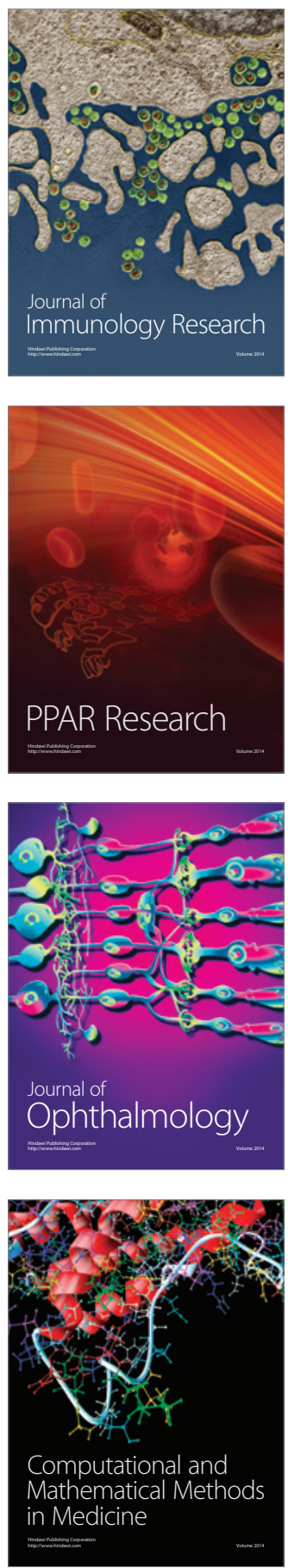

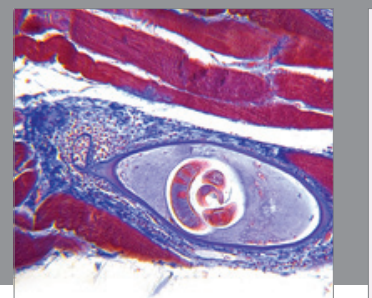

Gastroenterology

Research and Practice
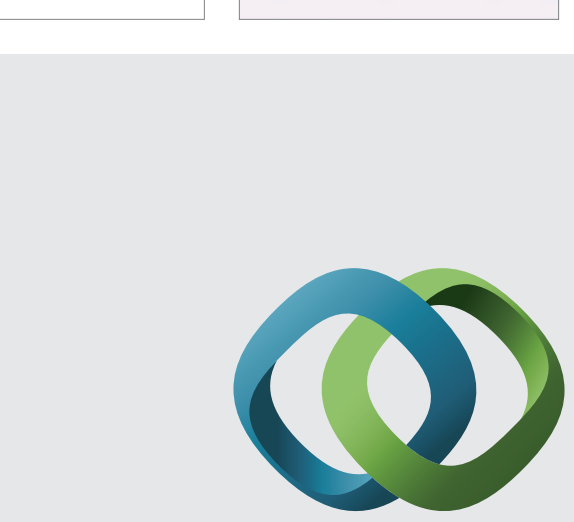

\section{Hindawi}

Submit your manuscripts at

http://www.hindawi.com
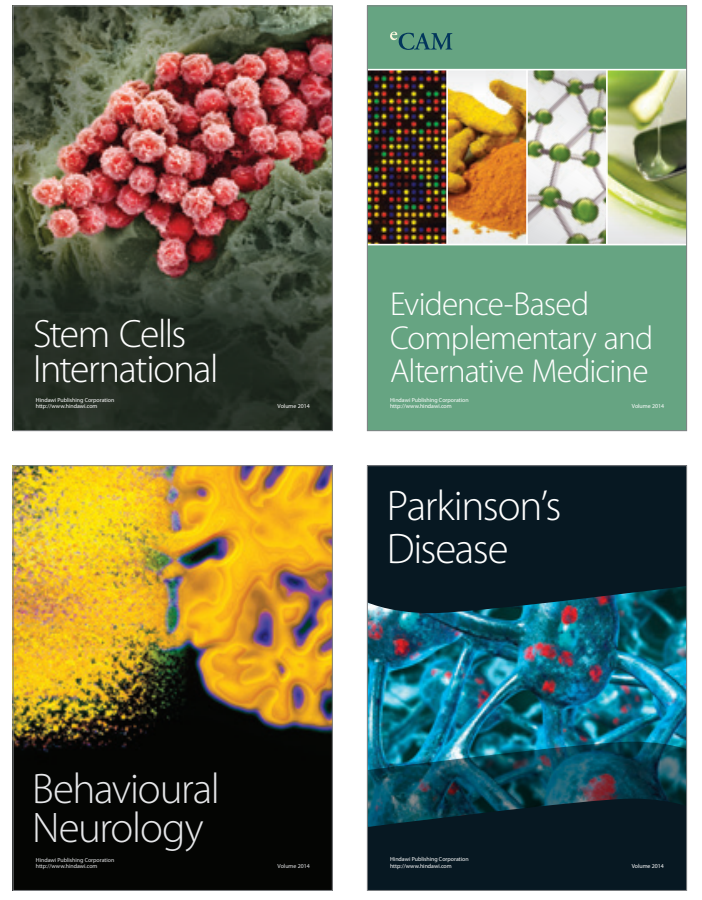
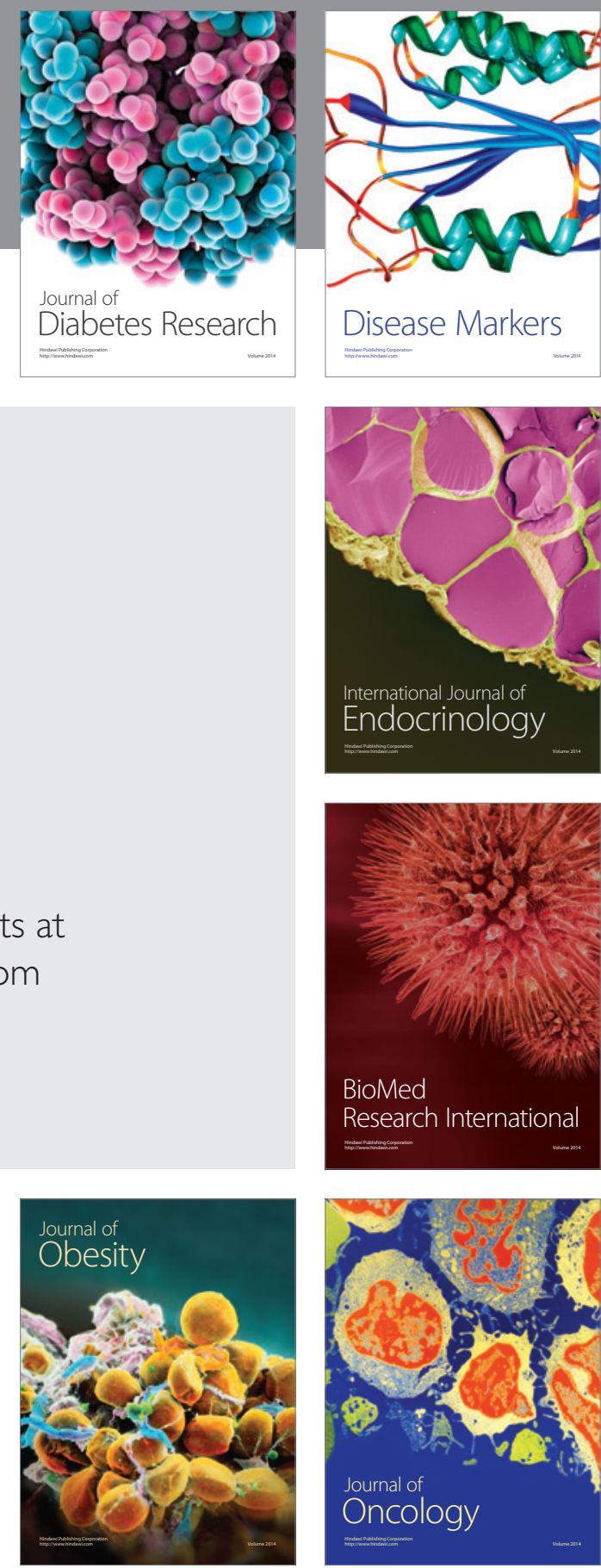

Disease Markers
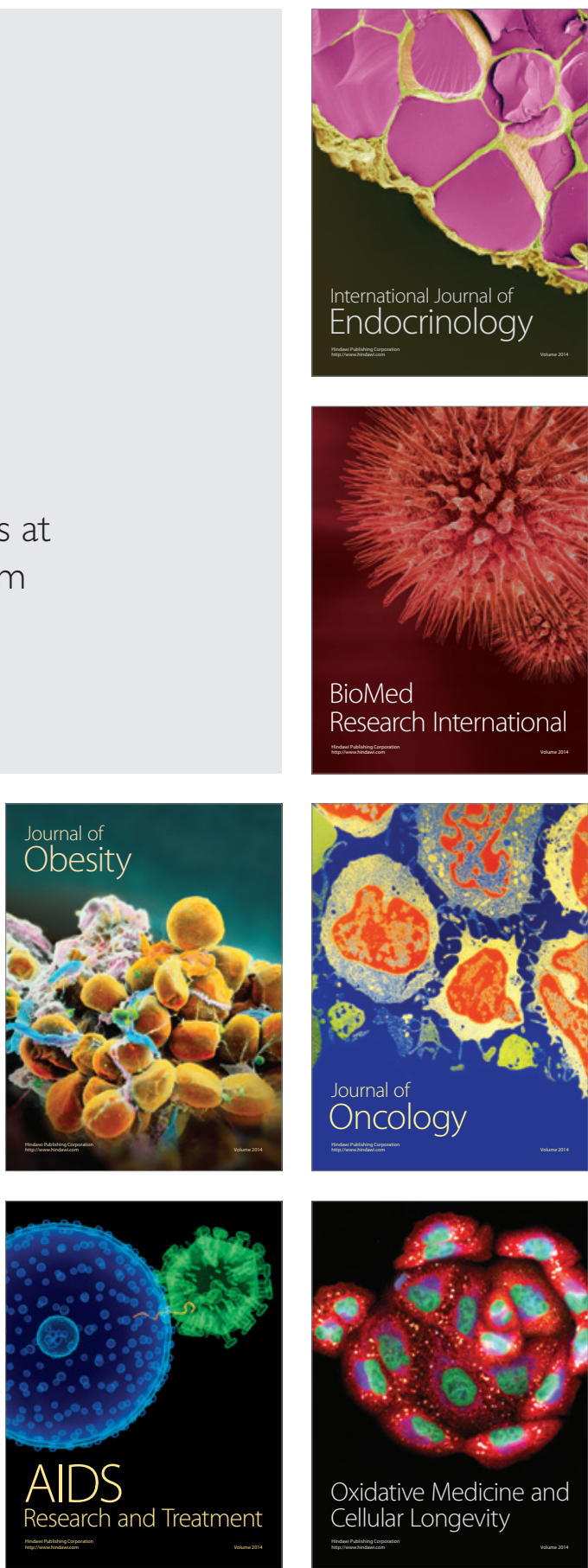